\title{
P15. A genetic mouse model to identify the role of the immune adapter protein MyD88 in colorectal cancer
}

\author{
A Holtorf ${ }^{*}$, B Holzmann, KP Janssen \\ From 1st Immunotherapy of Cancer Conference (ITOC1) \\ Munich, Germany. 12-14 March 2014
}

\section{Background}

Pattern recognition receptors from the Toll-like receptor (TLR) family are pivotal components of innate immunity, and have been shown to contribute to colon cancer formation. However, the molecular and cellular mechanisms underlying TLR-signaling in colon cancer remain unclear. The adapter protein Myeloid-differentiation factor 88 (MyD88) is shared between several TLRs and the Interleukin-1 receptor family. MyD88-deficiency protects mice from intestinal cancer formation in genetic models for colon cancer. The genetic mouse model presented here allows tissue-specific expression of MyD88, and thereby the dissection of the complex interaction between tumour and immune system during intestinal carcinogenesis.

\section{Material and methods}

Insertion of an 'intron-gene-trap' flanked with loxP motifs into the first intron of the MyD88 gene locus leads to global inactivation of myD88 expression $\left(\mathrm{MyD} 88^{\mathrm{LSL}}\right)$, faithfully phenotyping a global gene knockout. Tissue-specific re-expression of MyD88 in mice is mediated based on the Cre-recombinase. Breeding of MyD88 ${ }^{\mathrm{LSL}}$ mice with LysMCre or pvillin-Cre mice leads to tissue-specific excision of the 'intron-gene-trap', retaining endogenous regulation of gene expression. MyD88 expression and successful reconstitution of TLR-signaling was detected in either myeloid cells $\left(\mathrm{MyD}^{\mathrm{MYEL}}{ }^{\mathrm{M}}\right.$ ) or intestinal epithelial cells (MyD88 ${ }^{\mathrm{IEC}}$ ). Subsequently, these animals were mated with $\mathrm{Apc}^{1638 \mathrm{~N} /+}$ mice, an established genetic mouse model for human colon cancer.

\section{Results}

Global MyD88 deficiency dramatically decreased tumour incidence and aggressiveness in $\mathrm{Apc}^{1638 \mathrm{~N} /+}$ mice. Re-expression of MyD88 in intestinal epithelial cells only partially restored tumor formation. On the other hand, reconstitution of MyD88 expression in myeloid cells triggered tumour development virtually indistinguishable from parental $\mathrm{Apc}^{1638 \mathrm{~N} /+}$ mice. Activation of the canonical Wnt signaling pathway, induced by loss of function of Apc, was independent of MyD88. In contrast, MyD88 expression was required for full activation of MAPK/ERK signaling in intestinal epithelial cells. Furthermore, our results suggest a pro-tumorigenic function for the pro-inflammatory cytokines IL-1beta and IL-6, which were produced in a MyD88-dependent fashion by myeloid cells.

\section{Conclusions}

MyD88-mediated signaling has pro-tumorigenic effects in both IECs and in myeloid cells, but via different mechanisms. Moreover, MyD88 function in myeloid cells is crucial for intestinal tumour development, and its inhibition may form a promising therapeutic strategy.

Published: 12 March 2014

doi:10.1186/2051-1426-2-S2-P6

Cite this article as: Holtorf et al.: P15. A genetic mouse model to identify the role of the immune adapter protein MyD88 in colorectal cancer. Journal for ImmunoTherapy of Cancer 2014 2(Suppl 2):P6. 